\title{
Symbol alphabets from plabic graphs III: $n=9$
}

\author{
J. Mago, ${ }^{a}$ A. Schreiber, ${ }^{b}$ M. Spradlin, ${ }^{a, c}$ A. Yelleshpur Srikant ${ }^{a}$ and A. Volovich ${ }^{a}$ \\ ${ }^{a}$ Department of Physics, Brown University, \\ Providence, RI 02912, U.S.A. \\ ${ }^{b}$ The Mathematical Institute, University of Oxford, \\ Woodstock Road, OX2 6GG, U.K. \\ ${ }^{c}$ Brown Theoretical Physics Center, Brown University, \\ Providence, RI 02912, U.S.A. \\ E-mail: jorge_mago@brown.edu, anders_schreiber@alumni.brown.edu, \\ marcus_spradlin@brown.edu, akshay_yelleshpur_srikant@brown.edu, \\ anastasia_volovich@brown.edu
}

Abstract: Symbol alphabets of $n$-particle amplitudes in $\mathcal{N}=4$ super-Yang-Mills theory are known to contain certain cluster variables of $\mathrm{G}(4, n)$ as well as certain algebraic functions of cluster variables. In this paper we solve the $C Z=0$ matrix equations associated to several cells of the totally non-negative Grassmannian, combining methods of arXiv:2012.15812 for rational letters and arXiv:2007.00646 for algebraic letters. We identify sets of parameterizations of the top cell of $\mathrm{G}_{+}(5,9)$ for which the solutions produce all of (and only) the cluster variable letters of the 2-loop nine-particle NMHV amplitude, and identify plabic graphs from which all of its algebraic letters originate.

KEYwords: Scattering Amplitudes, Supersymmetric Gauge Theory

ArXiv EPrint: 2106.01406 


\section{Contents}

1 Introduction 1

2 Rational letters $\quad 2$

$2.1 n=8$ extended rational alphabet 2

$2.2 n=9$ rational alphabet 5

3 Algebraic letters $\quad 6$

$3.1 n=9$ two-loop NMHV algebraic symbol letters $\quad 7$

$3.2 n=9$ algebraic letters from plabic graphs 8

\section{Introduction}

The fact that cluster algebras [1-6] govern the symbol alphabets [7] of multiloop $n$-particle amplitudes in planar maximally supersymmetric Yang-Mills (SYM) theory is by now wellestablished for $n=6,7$ [8] (see [9] for a review of recent progress on the computation of these amplitudes via bootstrap). Starting at $n=8$ qualitatively new features arise, which have been studied via several different approaches (see for example [10-18]).

In this paper we continue the program outlined in [13-15], which is based on the observation that symbol letters of SYM theory seem to naturally emerge from certain plabic graphs [19] (or equivalently, Yangian invariants). Specifically, if $Z$ is an $n \times 4$ momentum twistor matrix parameterizing the kinematic data for an $n$-particle scattering process, and if $C$ is a $k \times n$ matrix parameterizing a $4 k$-dimensional cell of the totally non-negative Grassmannian $\mathrm{G}_{+}(k, n)$ [20], then solving the matrix equations $C Z=0$ [21, 22] sets the parameters of $C$ to various rational or algebraic functions of Plücker coordinates on $\mathrm{G}(4, n)$ that often turn out to be products of symbol letters of amplitudes.

In $[13,14]$ an example for $(k, n)=(2,8)$ was considered that precisely reproduces all of the 18 algebraic symbol letters known to appear in the 2-loop eight-particle NMHV amplitude [23]. At the same time it was pointed out that if the cell parameterized by $C$ is not the top cell (i.e., the one with dimension $k(n-k)$ ), then one generally encounters rational quantities that are not expressible in terms of cluster variables. On the other hand, in [15] it was shown that for any cluster parameterization of the top cell (not necessarily one associated to a plabic graph), this procedure will only give cluster variables.

Here our focus is on the case $n=9$, where the most up-to-date symbol alphabet information comes from the computation of the two-loop NMHV amplitude [24]. We show how to obtain all known $n=9$ symbol letters from cluster parameterizations of cells of $\mathrm{G}_{+}(k, 9)$. First, we provide an explicit list of cluster parameterizations of the top cell of $\mathrm{G}_{+}(5,9)$ which collectively provide all 531 of the $n=9$ rational letters found in [24] (and no additional letters). Second, we identify a cyclic class of parameterizations of cells of 
$\mathrm{G}_{+}(3,9)$ which collectively provide all 99 of the $n=9$ algebraic letters, together with a few additional algebraic quantities.

As already acknowledged in $[13,15]$, we do not as of yet have a "theory" to explain the pattern of which cells are associated to cluster variables (or algebraic functions thereof) that are actually observed to appear in amplitudes. Instead, we view our work as providing some kind of "phenomenological" data in the hope that future work will be able to shed more light on this interesting problem.

\section{$2 \quad$ Rational letters}

\section{$2.1 n=8$ extended rational alphabet}

To date, a total of 180 rational letters, all of which are cluster variables of $\mathrm{G}(4,8)$, are known to appear in the eight-particle amplitudes of SYM theory. These letters are tabulated in [23]. By studying a certain fan one can naturally associate to the tropical positive Grassmannian (or, equivalently, its dual polytope), [10-12] encountered a larger list of cluster variables that includes these 180, together with 100 more. These additional variables may appear in the symbols of eight-point amplitudes that have not yet been computed. We call this collection of 280 cluster variables the $n=8$ extended rational alphabet; it consists of

- 68 four-brackets of the form $\langle a a+1 b c\rangle$,

- 8 cyclic images of $\langle 12 \overline{4} \cap \overline{7}\rangle$,

- 40 cyclic images of $\langle 1(23)(45)(78)\rangle,\langle 1(23)(56)(78)\rangle,\langle 1(28)(34)(56)\rangle,\langle 1(28)(34)(67)\rangle$, $\langle 1(28)(45)(67)\rangle$,

- 48 dihedral images of $\langle 1(23)(45)(67)\rangle,\langle 1(23)(45)(68)\rangle,\langle 1(28)(34)(57)\rangle$,

- 8 cyclic images of $\langle\overline{2} \cap(245) \cap \overline{8} \cap(856)\rangle$,

- 8 distinct images of $\langle\overline{2} \cap(245) \cap \overline{6} \cap(681)\rangle$,

- 16 dihedral images of $\langle\langle 12345678\rangle\rangle$,

- 2 letters, $\langle 1357\rangle$ and $\langle 2468\rangle$,

- 8 cyclic images of $\langle 1(23)(46)(78)\rangle$,

- 16 dihedral images of $\langle 1(27)(34)(56)\rangle$,

- 2 cyclic images of $\langle\overline{2} \cap \overline{4} \cap \overline{6} \cap \overline{8}\rangle$,

- 8 cyclic images of $\langle\overline{2} \cap(246) \cap \overline{6} \cap \overline{8}\rangle$,

- 32 dihedral images of $\langle\langle 12435678\rangle\rangle,\langle\langle 12436578\rangle\rangle$,

- 16 dihedral images of $\langle 1234\rangle\langle 1678\rangle\langle 2456\rangle-\langle 1267\rangle\langle 1348\rangle\langle 2456\rangle+\langle 1248\rangle\langle 1267\rangle\langle 3456\rangle$. 
Here $\langle a b c d\rangle$ are Plücker coordinates on $\mathrm{G}(4, n)$ and we define

$$
\begin{aligned}
\bar{a} & \equiv(a-1 a a+1), \\
\langle a(b c)(d e)(f g)\rangle & \equiv\langle a b d e\rangle\langle a c f g\rangle-\langle a c d e\rangle\langle a b f g\rangle, \\
\langle a, b, c,(d e) \cap(f g h)\rangle \equiv\langle a b c d\rangle\langle e f g h\rangle-\langle a b c e\rangle\langle d f g h\rangle, & \\
\langle x, y,(a b c) \cap(\text { def })\rangle & \equiv\langle x a b c\rangle\langle y d e f\rangle-\langle y a b c\rangle\langle x d e f\rangle, \\
\langle\langle a b c d e f g h\rangle\rangle \equiv & \langle a b c d\rangle\langle a b e f\rangle\langle\text { degh }\rangle-\langle a b d e\rangle\langle a b e f\rangle\langle c d g h\rangle \\
& +\langle a b d e\rangle\langle a b g h\rangle\langle c d e f\rangle, \\
\langle\bar{x} \cap(a b c) \cap \bar{y} \cap(\text { def })\rangle \equiv & \langle a,(b c) \cap \bar{x}, d,(e f) \cap \bar{y}\rangle .
\end{aligned}
$$

We know from [15] that for any cluster parameterization $C$ of the top cell of $\mathrm{G}_{+}(4,8)$, solving $C Z=0$ expresses the parameters of $C$ in terms of products of powers of $\mathrm{G}(4,8)$ cluster variables. Our aim is to identify a set of parameterizations that collectively involve precisely the 280 letters of the extended rational alphabet (and no other letters).

We begin by taking $C$ to be the boundary measurement of the plabic graph shown in figure 1 (see $[13,15]$ for more details on our conventions). Then the solution to $C Z=0$ is given by

$$
\begin{aligned}
& f_{0}=-\frac{\langle 1234\rangle}{\langle 2348\rangle}, \quad f_{1}=\frac{\langle 3458\rangle\langle 4567\rangle}{\langle 3456\rangle\langle 4578\rangle}, \quad f_{2}=\frac{\langle 2348\rangle\langle 3456\rangle\langle 4578\rangle}{\langle 2345\rangle\langle 3478\rangle\langle 4568\rangle}, \\
& f_{3}=\frac{\langle 3478\rangle\langle 4568\rangle}{\langle 3458\rangle\langle 4678\rangle}, \quad f_{4}=\frac{\langle 1238\rangle\langle 2345\rangle\langle 3478\rangle}{\langle 1234\rangle\langle 2378\rangle\langle 3458\rangle}, \quad f_{5}=\frac{\langle 2378\rangle\langle 3458\rangle\langle 4678\rangle}{\langle 2348\rangle\langle 3678\rangle\langle 4578\rangle}, \\
& f_{6}=\frac{\langle 3678\rangle\langle 4578\rangle}{\langle 3478\rangle\langle 5678\rangle}, \quad f_{7}=\frac{\langle 1278\rangle\langle 2348\rangle\langle 3678\rangle}{\langle 1238\rangle\langle 2678\rangle\langle 3478\rangle}, \quad f_{8}=\frac{\langle 2678\rangle\langle 3478\rangle}{\langle 2378\rangle\langle 4678\rangle}, \\
& f_{9}=\frac{\langle 1678\rangle\langle 2378\rangle}{\langle 1278\rangle\langle 3678\rangle}, \quad f_{10}=\frac{\langle 2678\rangle}{\langle 1678\rangle}, \quad f_{11}=\frac{\langle 3678\rangle}{\langle 2678\rangle}, \\
& f_{12}=\frac{\langle 4678\rangle}{\langle 3678\rangle}, \quad f_{13}=-\frac{\langle 4568\rangle}{\langle 4567\rangle}, \quad f_{14}=-\frac{\langle 4578\rangle}{\langle 4568\rangle}, \\
& f_{15}=-\frac{\langle 4678\rangle}{\langle 4578\rangle}, \quad f_{16}=\frac{\langle 5678\rangle}{\langle 4678\rangle} .
\end{aligned}
$$

By drawing the dual quiver (with arrows clockwise around white vertices and counterclockwise around black vertices) and reading off the adjacency matrix, we can mutate the face variables according to the cluster $\mathcal{X}$-variable mutation rules [25]

$$
f_{k}^{\prime}= \begin{cases}f_{k}^{-1} & i=k \\ f_{i}\left(1+f_{k}^{-\operatorname{sgn}\left(b_{i, k}\right)}\right)^{-b_{i, k}} & i \neq k\end{cases}
$$

where $b_{i, j}$ is the adjacency matrix of the dual quiver. Under mutations, the adjacency matrix transforms as

$$
b_{i, j}^{\prime}=\left\{\begin{array}{llr}
-b_{i, j} & & k \in\{i, j\} \\
b_{i, j} & k \notin\{i, j\} \text { and } b_{i, k} b_{k, j} \leq 0 ; \\
b_{i, j}+\left|b_{i, k}\right| b_{k, j} & k \notin\{i, j\} \text { and } b_{i, k} b_{k, j}>0
\end{array}\right.
$$




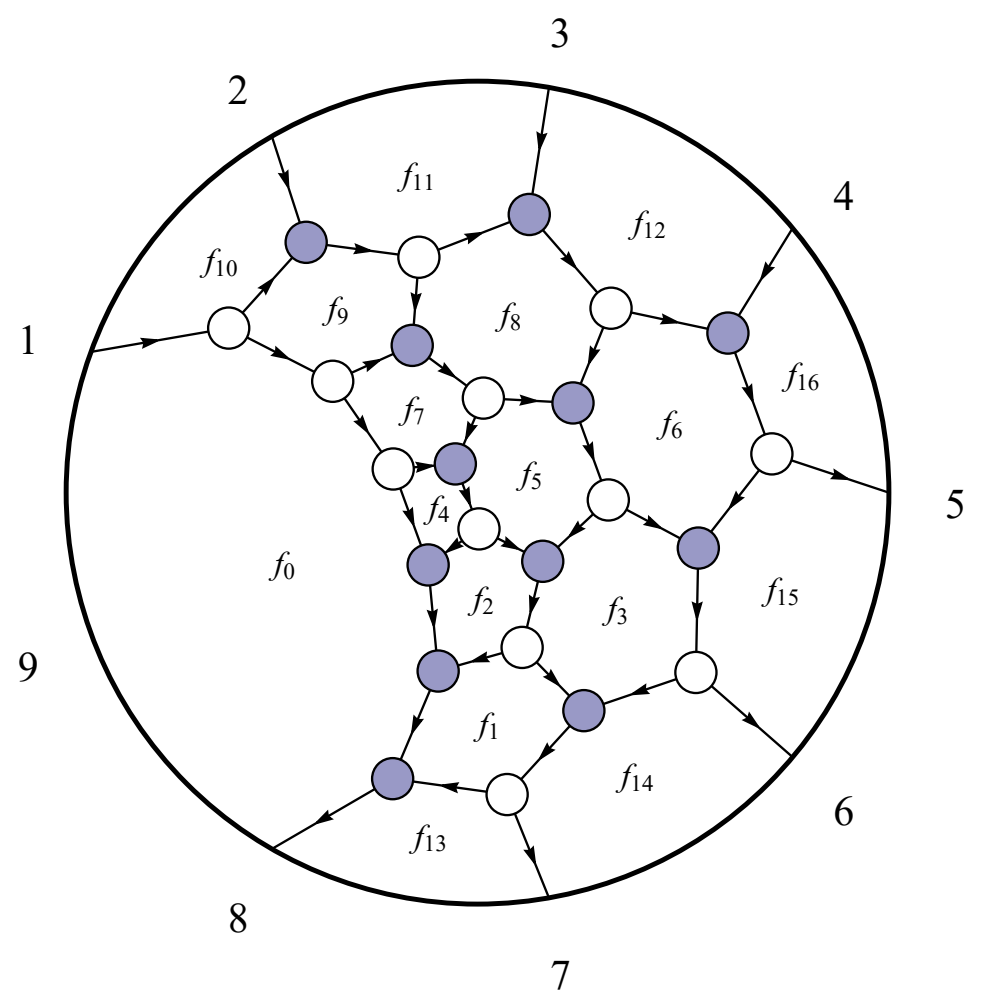

Figure 1. A plabic graph associated to the top cell of $\mathrm{G}_{+}(4,8)$.

We perform sequences of mutations on internal faces (external faces are considered frozen) and collect all monomial factors that appear in the mutated face variables. We then find a minimal set of mutation sequences for which the mutated face variables collectively contain the entire 280 letter extended rational alphabet (mod cyclic permutations of external labels), and only letters from that alphabet. Note that the cluster algebra associated with the dual quiver of the $\mathrm{G}_{+}(4,8)$ top cell is of infinite type, and we only search far enough to find minimal length mutation sequences that suffice to produce the entire 280-letter alphabet.

We find that considering all mutation sequences of up to length 5 is sufficient, and in particular we find 13 clusters that are sufficient to generate the entire 280-letter $n=8$ extended rational alphabet (mod cyclic rotations of external labels). These clusters are obtained from the following 13 mutation sequences:

$$
\begin{aligned}
& \{\{4,7,8,3,6\},\{5,7,9,8,2\},\{5,8,3,1,2\},\{6,8,7,4,2\}, \\
& \{7,1,2,5,6\},\{7,2,3,6,5\},\{7,4,2,3,6\},\{7,5,6,2,1\}, \\
& \{8,3,5,2,4\},\{8,4,5,1,3\},\{8,6,3,2,4\},\{9,1,2,5,7\},\{9,8,5,3,1\}\},
\end{aligned}
$$

where the sequences should be read as: $\{a, b, c, \ldots\}$ : mutate on the node $f_{a}$, then mutate on $f_{b}$, and then mutate on $f_{c}$, etc. It is important to emphasize that this set of minimal length mutational sequences is not unique. Also, note that at intermediate steps between 
the initial cluster and the final 13 clusters obtained at the end of these sequences, one can encounter additional cluster variables not contained in the 280-letter alphabet.

\section{$2.2 n=9$ rational alphabet}

To date, a total of 531 rational letters, all of which are cluster variables of G(4,9), are known to appear in the nine-particle amplitudes of SYM theory. These letters are tabulated in [24] and consist of:

- 13 cyclic classes of $\langle 12 k l\rangle$ for $3 \leq k<l \leq 8$ but $(k, l) \neq(6,7),(7,8)$;

- 7 cyclic classes of $\langle 12(i j k) \cap(\operatorname{lmn})\rangle$ for $3 \leq i<j<k<l<m<n \leq 9$;

- 8 cyclic classes of $\langle\overline{2} \cap(245) \cap \overline{6} \cap(691)\rangle,\langle\overline{2} \cap(346) \cap \overline{6} \cap(892)\rangle,\langle\overline{2} \cap(346) \cap \overline{2} \cap(782)\rangle$, $\langle\overline{2} \cap(245) \cap \overline{7} \cap(791)\rangle,\langle\overline{2} \cap(245) \cap(568) \cap \overline{8}\rangle,\langle\overline{2} \cap(245) \cap(569) \cap \overline{9}\rangle,\langle\overline{2} \cap(245) \cap(679) \cap \overline{9}\rangle$, $\langle\overline{2} \cap(256) \cap(679) \cap \overline{9}\rangle ;$

- 10 cyclic classes of $\langle 1(i i+1)(j j+1)(k k+1)\rangle$ for $2 \leq i, i+1<j, j+1<k \leq 8$;

- 6 cyclic classes $\langle 1(2 i)(j j+1)(k 9)\rangle$ for $3 \leq i<j, j+1<k \leq 8$, but $(i, k) \neq$ $(3,8),(4,7)$;

- 14 cyclic classes of $\langle 1(29)(i j)(k k+1)\rangle$ for $3<i<j \leq 8,3 \leq k \leq i-2$ or $j+1 \leq k \leq 7$;

- 1 cyclic class of $\langle 1,(56) \cap \overline{3},(78) \cap \overline{3}, 9\rangle$.

In this section, we derive this alphabet from plabic and non-plabic parameterizations of the top cell in $\mathrm{G}_{+}(5,9)$ by analyzing sequences of mutations on the dual quiver to the initial plabic parameterizations of the top cell, corresponding to the plabic graph shown in figure 2. Taking $C$ to be the boundary measurement of this graph, we find that the solution to $C Z=0$ is given by

$$
\begin{aligned}
& f_{0}=-\frac{\langle 1234\rangle}{\langle 2349\rangle}, \quad f_{1}=\frac{\langle 4569\rangle\langle 5678\rangle}{\langle 4567\rangle\langle 5689\rangle}, \quad f_{2}=\frac{\langle 3459\rangle\langle 4567\rangle\langle 5689\rangle}{\langle 3456\rangle\langle 4589\rangle\langle 5679\rangle}, \\
& f_{3}=\frac{\langle 4589\rangle\langle 5679\rangle}{\langle 4569\rangle\langle 5789\rangle}, \quad f_{4}=\frac{\langle 2349\rangle\langle 3456\rangle\langle 4589\rangle}{\langle 2345\rangle\langle 3489\rangle\langle 4569\rangle}, \quad f_{5}=\frac{\langle 3489\rangle\langle 4569\rangle\langle 5789\rangle}{\langle 3459\rangle\langle 4789\rangle\langle 5689\rangle}, \\
& f_{6}=\frac{\langle 4789\rangle\langle 5689\rangle}{\langle 4589\rangle\langle 6789\rangle}, \quad f_{7}=\frac{\langle 1239\rangle\langle 2345\rangle\langle 3489\rangle}{\langle 1234\rangle\langle 2389\rangle\langle 3459\rangle}, \quad f_{8}=\frac{\langle 2389\rangle\langle 3459\rangle\langle 4789\rangle}{\langle 2349\rangle\langle 3789\rangle\langle 4589\rangle}, \\
& f_{9}=\frac{\langle 3789\rangle\langle 4589\rangle}{\langle 3489\rangle\langle 5789\rangle}, \quad f_{10}=\frac{\langle 1289\rangle\langle 2349\rangle\langle 3789\rangle}{\langle 1239\rangle\langle 2789\rangle\langle 3489\rangle}, \quad f_{11}=\frac{\langle 2789\rangle\langle 3489\rangle}{\langle 2389\rangle\langle 4789\rangle}, \\
& f_{12}=\frac{\langle 1789\rangle\langle 2389\rangle}{\langle 1289\rangle\langle 3789\rangle}, \quad f_{13}=\frac{\langle 2789\rangle}{\langle 1789\rangle}, \quad f_{14}=\frac{\langle 3789\rangle}{\langle 2789\rangle}, \\
& f_{15}=\frac{\langle 4789\rangle}{\langle 3789\rangle}, \quad f_{16}=\frac{\langle 5789\rangle}{\langle 4789\rangle}, \quad f_{17}=-\frac{\langle 5679\rangle}{\langle 5678\rangle}, \\
& f_{18}=-\frac{\langle 5689\rangle}{\langle 5679\rangle}, \quad f_{19}=-\frac{\langle 5789\rangle}{\langle 5689\rangle}, \quad f_{20}=\frac{\langle 6789\rangle}{\langle 5789\rangle} .
\end{aligned}
$$




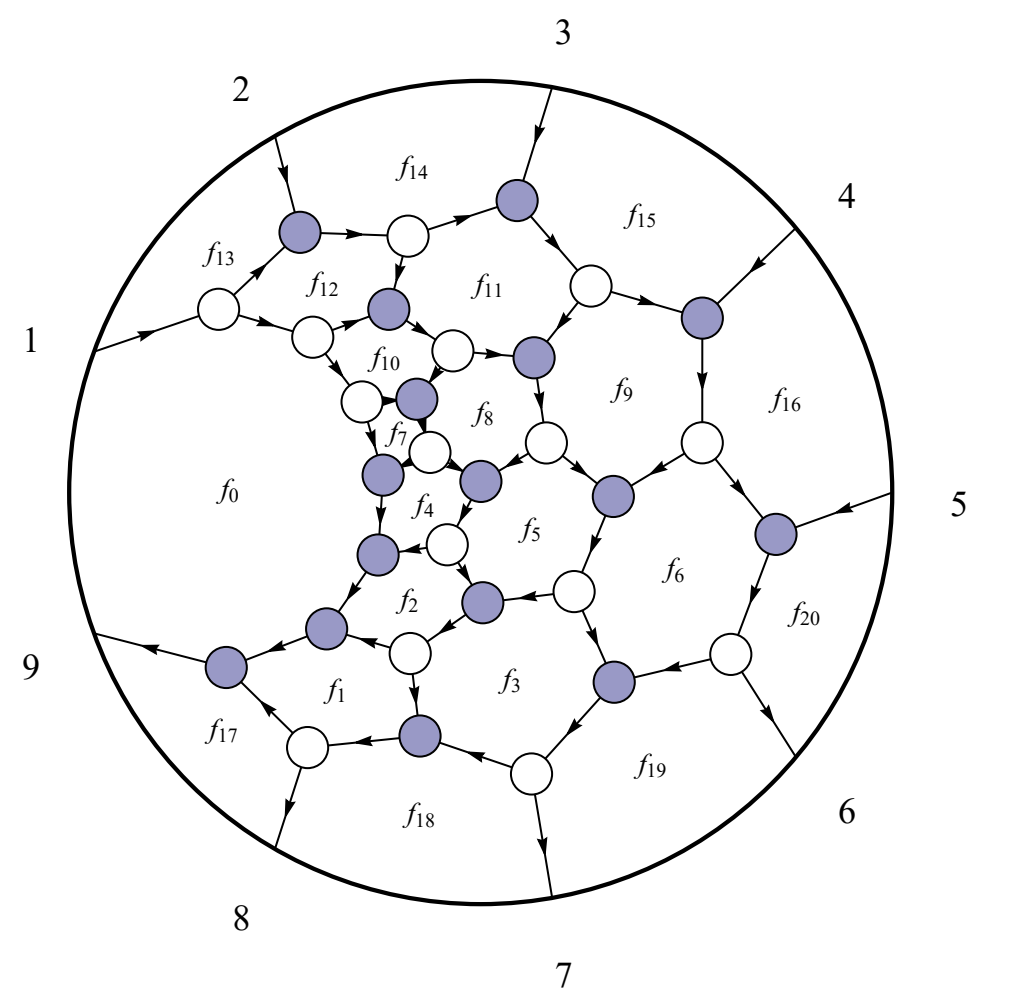

Figure 2. A plabic graph associated to the top cell of $\mathrm{G}_{+}(5,9)$.

We find that mutation sequences of up to length 8 are sufficient to generate the entire $n=9$ rational symbol alphabet (mod cyclic rotation of the external labels). In particular, the 15 clusters reached from the initial quiver described above by the mutation sequences

$$
\begin{aligned}
& \{\{1,3,2,5,8,7,11,12\},\{1,5,2,10,8,10,12,11\},\{1,5,3,9,5,8,11,12\}, \\
& \{2,4,6,5,9,8,11,9\},\{2,4,6,9,5,8,12,10\},\{2,4,7,8,11,8,12,10\}, \\
& \{3,1,6,5,8,9,11,12\},\{3,4,2,5,8,4,7,10\},\{4,2,8,9,8,12,10,11\}, \\
& \{5,6,3,7,11,10,8,12\},\{9,4,2,5,1,3,2\},\{9,11,6,4,8,7,10\}, \\
& \{10,7,5,3,2,4,5\},\{11,6,3,2,4,7,10\},\{12,10,1,2,4,8,5\}\},
\end{aligned}
$$

suffice to generate the entire 531-letter $n=9$ rational symbol alphabet ( $\bmod$ cyclic rotations of the external labels). These 15 clusters contain only letters from this symbol alphabet. Again we note that this set of minimal length mutation sequences is not unique, and that cluster variables outside the 531-letter alphabet may be encountered at intermediate steps along these sequences.

\section{$3 \quad$ Algebraic letters}

In this section we show how to obtain the algebraic letters of the $n=9$ two-loop NMHV symbol alphabet [24] by solving $C Z=0$ for plabic parameterizations of non-top cells of $\mathrm{G}_{+}(4,9)$. This generalizes the corresponding analysis for $n=8$ carried out in $[13,14]$. 


\section{$3.1 n=9$ two-loop NMHV algebraic symbol letters}

In [24] it was found that 99 multiplicatively independent algebraic symbol letters appear in the symbol of the two-loop nine-particle NMHV amplitude. All algebraic letters of twoloop NMHV amplitudes trace their origin to the one-loop four-mass box integral. Here we recall some definitions useful for expressing these letters:

$$
\begin{aligned}
u_{a b c d} & \equiv \frac{\langle a-1 a b-1 b\rangle\langle c-1 c d-1 d\rangle}{\langle a-1 a c-1 c\rangle\langle b-1 b d-1 d\rangle}, \quad v_{a b c d} \equiv \frac{\langle b-1 b c-1 c\rangle\langle a-1 a d-1 d\rangle}{\langle a-1 a c-1 c\rangle\langle b-1 b d-1 d\rangle}, \\
\Delta_{a b c d} & \equiv \sqrt{\left(1-u_{a b c d}-v_{a b c d}\right)^{2}-4 u_{a b c d} v_{a b c d}}, \\
z_{a b c d} & \equiv \frac{1}{2}\left(1+u_{a b c d}-v_{a b c d}+\Delta_{a b c d}\right), \quad \bar{z}_{a b c d} \equiv \frac{1}{2}\left(1+u_{a b c d}-v_{a b c d}-\Delta_{a b c d}\right) .
\end{aligned}
$$

We will also define

$$
\begin{aligned}
x_{a b c d}^{a} & =\frac{\langle\bar{d},(c-1, c) \cap(a, b-1, b)\rangle}{\langle\bar{d}, a\rangle\langle b-1, b, c-1, c\rangle}, \\
x_{a b c d}^{b} & =\frac{\langle\bar{d},(c-1, c) \cap(a-1, a, b)\rangle}{\langle\bar{d},(a-1, a) \cap(b, c-1, c)\rangle}, \\
x_{a b c d}^{c} & =\frac{\langle\bar{d}, c\rangle\langle a-1, a, b-1, b\rangle}{\langle\bar{d},(a-1, a) \cap(b-1, b, c)\rangle},
\end{aligned}
$$

where $x_{a b c d}^{b-1}, x_{a b c d}^{c-1}$ differ by exchanging $a \leftrightarrow a-1$ when the superscript is $a-1$, exchanging $b \leftrightarrow b-1$ when the superscript is $b-1$, and so on. With this, we can define two classes of algebraic symbol letters

$$
\mathcal{X}_{a b c d}^{\star} \equiv \frac{\left(x_{a b c d}^{\star}+1\right)^{-1}-\bar{z}_{d a b c}}{\left(x_{a b c d}^{\star}+1\right)^{-1}-z_{d a b c}}, \quad \tilde{\mathcal{X}}_{a b c d}^{\star} \equiv \frac{\left(x_{a b c(d-1)}^{\star}+1\right)^{-1}-z_{d a b c}}{\left(x_{a b c(d-1)}^{\star}+1\right)^{-1}-\bar{z}_{d a b c}},
$$

where the star $\star$ corresponds to the six choices $a-1, a, b-1, b, c-1, c$ of the superscript of $x_{a b c d}^{\star}$. We note that $\mathcal{X}_{a b c d}^{\star}, \mathcal{X}_{b c d a}^{\star}, \mathcal{X}_{c d a b}^{\star}$ and $\mathcal{X}_{d a b c}^{\star}$ all depend on the same square root $\Delta_{a b c d}$. With this, we have a total of $4 \times 2 \times 6=48$ algebraic letters depending on each $\Delta_{a b c d}$ from $\mathcal{X}_{a b c d}^{\star}$ and $\tilde{\mathcal{X}}_{a b c d}^{\star}$. In addition to these letters, there are two more letters depending on $\Delta_{a b c d}$

$$
X_{a b c d}=\frac{z_{a b c d}}{\bar{z}_{a b c d}}, \quad \text { and } \quad \tilde{X}_{a b c d}=\frac{1-z_{a b c d}}{1-\bar{z}_{a b c d}},
$$

bringing us to a grand total of 50 algebraic letters depending on $\Delta_{a b c d}$ in the most general case. However, in cases where $0 \leq m \leq 4$ of the corners of the four-mass box (from which these letters originate) contain only two particles, the number of independent letters containing $\Delta_{a b c d}$, is reduced to $50-2 \mathrm{~m}$. In addition, there are 33 multiplicative relations between the algebraic symbol letters of (3.5) and (3.6), meaning that the number of independent letters containing $\Delta_{a b c d}$ is reduced to $17-2 m$. Thus in the nine-particle case, where we always have four-mass boxes with three corners containing two particles and one containing three, we have $m=3$ and thus $17-6=11$ letters for each $\Delta_{a b c d}$. There are nine different square roots at $n=9$, so there are in total $11 \times 9=99$ independent algebraic symbol letters at $n=9$. 


\section{$3.2 n=9$ algebraic letters from plabic graphs}

At $n=9$, there are two cyclic classes of positroid cells with intersection number 2 and dimension $4 k$. We recall from [13] that the latter condition is necessary for $C Z=0$ to admit solutions for generic $Z$, and the former condition is necessary for the solution to involve algebraic functions (and specifically, square roots). These two classes of cells are represented by the decorated permutations

$$
\{2,6,5,8,7,10,9,13,12\} \text {, and }\{2,6,4,8,7,10,9,12,14\} \text {. }
$$

Solutions to $C Z=0$ from the first class of positroid cells above do not yield square roots of the type found in (3.5) and (3.6), so we focus on the second class. In the second class we find it computationally convenient to work with the cyclic representative $\{3,6,8,5,9,7,11,10,13\}$, which is associated to the plabic graph shown in figure 3 .

Solving $C Z=0$, and picking one of the two solutions (the other is obtained by conjugating all roots), yields the following result in terms of the algebraic letters given in (3.5) and (3.6):

$$
\begin{aligned}
& f_{0}=\sqrt{\frac{\langle 1239\rangle^{2}\langle 8(23)(45)(67)\rangle \tilde{\mathcal{X}}_{357}^{a} \mathcal{X}_{593}^{c}}{\langle 2389\rangle\langle 89(45) \cap(123)(67) \cap(123)\rangle \tilde{\mathcal{X}}_{5793}^{a-1} \mathcal{X}_{9357}^{c}}}, \quad f_{1}=\sqrt{\frac{\langle 2389\rangle\langle 4567\rangle\left(\tilde{\mathcal{X}}_{5733}^{a-1}\right)^{2} \tilde{\mathcal{X}}_{7935}^{a-1}}{\langle 2345\rangle\langle 6789\rangle\left(\mathcal{X}_{5793}^{c}\right)^{2} \mathcal{X}_{7935}^{c}\left(\tilde{\mathcal{X}}_{5793}^{c}\right)^{2}}}, \\
& f_{2}=\sqrt{\frac{\langle 1289\rangle^{2}\langle 2345\rangle\langle 9(23)(45)(67)\rangle \tilde{\mathcal{X}}_{9357}^{b-1}}{\langle 1239\rangle^{2}\langle 4589\rangle\langle 2(45)(67)(89)\rangle \tilde{\mathcal{X}}_{9357}^{a}}}, \quad \quad f_{3}=\sqrt{\frac{\langle 2389\rangle\langle 67(23) \cap(189)(45) \cap(189)\rangle \tilde{\mathcal{X}}_{7935}^{a} \mathcal{X}_{3579}^{c}}{\langle 1289\rangle^{2}\langle 3(45)(67)(89)\rangle}}, \\
& f_{4}=\sqrt{\frac{\langle 89(45) \cap(123)(67) \cap(123)\rangle \tilde{\mathcal{X}}_{5793}^{a-1} \tilde{\mathcal{X}}_{7935}^{a-1}}{\langle 2389\rangle\langle 9(23)(45)(67)\rangle \mathcal{X}_{5793}^{c} \mathcal{X}_{7935}^{c}}}, \quad f_{5}=\sqrt{\frac{\langle 2389\rangle\langle 2(45)(67)(89)\rangle \tilde{\mathcal{X}}_{9357}^{b}}{\langle 67(23) \cap(189)(45) \cap(189)\rangle \tilde{\mathcal{X}}_{7935}^{a} \tilde{\mathcal{X}}_{9357}^{b-1} \mathcal{X}_{3579}^{c}}}, \\
& f_{6}=\sqrt{\frac{\langle 4589\rangle\langle 2(45)(67)(89)\rangle \tilde{\mathcal{X}}_{5793}^{c} \mathcal{X}_{7935}^{c} \mathcal{X}_{5793}^{c}}{\langle 2389\rangle\langle 5(23)(67)(89)\rangle \tilde{\mathcal{X}}_{5793}^{-1} \tilde{\mathcal{X}}_{7935}^{-1} \tilde{\mathcal{X}}_{9357}^{b-1}}}, \quad f_{7}=\sqrt{\frac{\langle 9(23)(45)(67)\rangle \mathcal{X}_{935}^{c} \mathcal{X}_{357}^{c}}{\langle 8(23)(45)(67)\rangle \tilde{\mathcal{X}}_{7935}^{a-1} \tilde{\mathcal{X}}_{9357}^{a}}}, \\
& f_{8}=\sqrt{\frac{\langle 4589\rangle\langle 6(23)(45)(89)\rangle \mathcal{X}_{9357}^{a} \mathcal{X}_{5793}^{c} \mathcal{X}_{7935}^{c} \tilde{\mathcal{X}}_{5793}^{c}}{\langle 4567\rangle\langle 9(23)(45)(67)\rangle \tilde{\mathcal{X}}_{5793}^{a-1} \tilde{\mathcal{X}}_{9357}^{b}}}, \quad f_{9}=\sqrt{\frac{\langle 7(23)(45)(89)\rangle \tilde{\mathcal{X}}_{7935}^{a}}{\langle 6(23)(45)(89)\rangle \tilde{\mathcal{X}}_{7935}^{a-1}}}, \\
& f_{10}=\sqrt{\frac{\langle 6789\rangle\langle 4(23)(67)(89)\rangle \tilde{\mathcal{X}}_{935}^{a-1} \tilde{\mathcal{X}}_{357}^{b}}{\langle 4589\rangle\langle 7(23)(45)(89)\rangle \tilde{\mathcal{X}}_{7935}^{a} \tilde{\mathcal{X}}_{9357} \mathcal{X}_{5793}^{c} \mathcal{X}_{7935}^{c}}}, \quad f_{11}=\sqrt{\frac{\langle 3(45)(67)(89)\rangle \tilde{\mathcal{X}}_{9357}^{b-1}}{\langle 2(45)(67)(89)\rangle \tilde{\mathcal{X}}_{9357}^{b}}}, \\
& f_{12}=\sqrt{\frac{\langle 5(23)(67)(89)\rangle \tilde{X}_{3357}^{a} \mathcal{X}_{5793}^{c}}{\langle 4(23)(67)(89)\rangle}} .
\end{aligned}
$$

All of these involve the common square root $\sqrt{\Delta_{3579}}$ of four-mass box type. The other 8 square roots can be obtained by cyclic rotations of the external labels. The 13 face variables can be expressed in terms of a basis of 11 multiplicatively independent algebraic letters:

$$
\left\{\mathcal{X}_{3579}^{c}, \mathcal{X}_{5793}^{c}, \mathcal{X}_{7935}^{c}, \mathcal{X}_{9357}^{c}, \tilde{\mathcal{X}}_{5793}^{a-1}, \tilde{\mathcal{X}}_{5793}^{c}, \tilde{\mathcal{X}}_{7935}^{a-1}, \tilde{\mathcal{X}}_{7935}^{a}, \tilde{\mathcal{X}}_{9357}^{a}, \tilde{\mathcal{X}}_{9357}^{b-1}, \tilde{\mathcal{X}}_{9357}^{b}\right\} .
$$

Performing all possible mutations on the internal faces of the plabic graph in figure 3 , we find an additional 12 unique factors, which can be expressed in terms of (3.8) as

$$
\begin{array}{lll}
1+f_{0}, & 1+f_{1}, & 1+f_{2}, \\
1+f_{3}, & 1+\left(1+f_{2}\right) f_{0}, & 1+\left(1+f_{1}\right) f_{2}, \\
1+f_{2}\left(1+f_{3}\right), & 1+f_{0}+f_{0} f_{2}\left(1+f_{1}\right), & 1+f_{0}+f_{0} f_{2}\left(1+f_{3}\right), \\
1+\left(1+f_{1}\right) f_{2}\left(1+f_{3}\right), & 1+f_{0}+f_{0}\left(1+f_{1}\right) f_{2}\left(1+f_{3}\right), \\
1+f_{2}+f_{0}\left(1+\left(1+f_{1}\right) f_{2}\right)\left(1+f_{2}\left(1+f_{3}\right)\right) .
\end{array}
$$




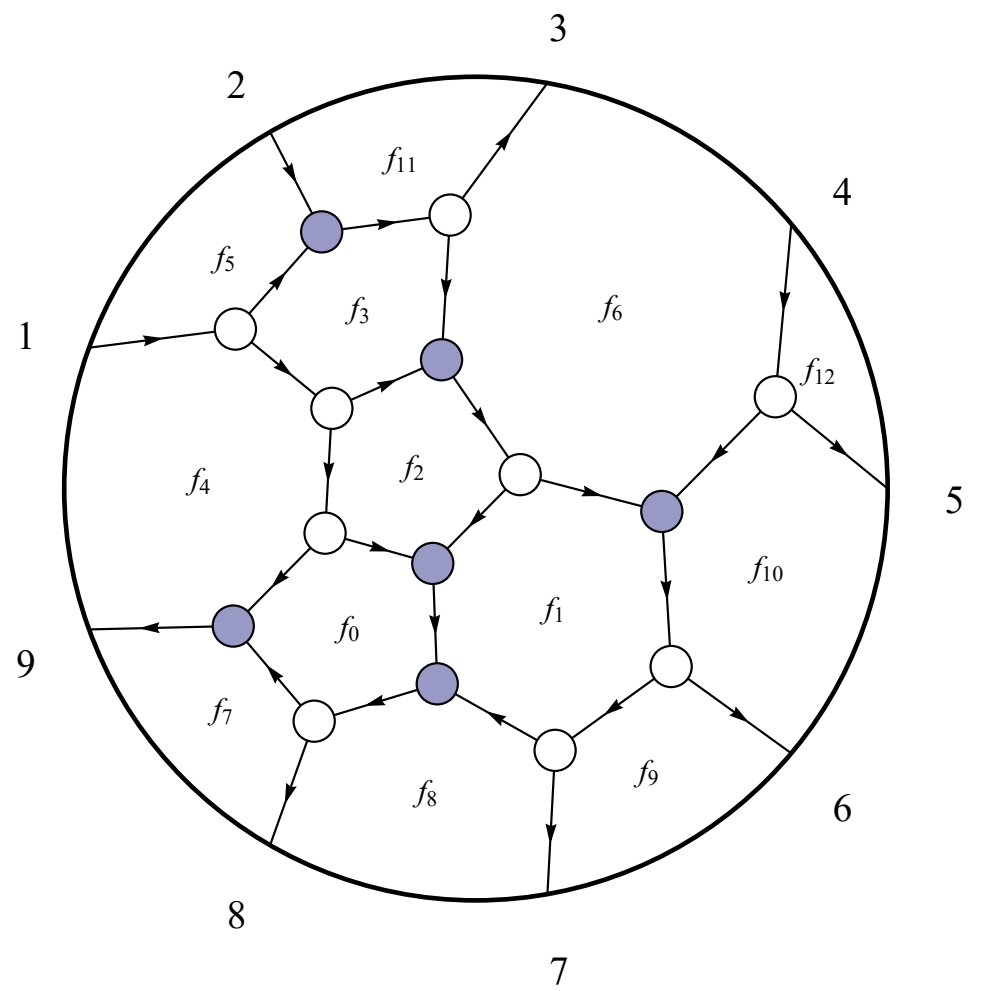

Figure 3. Plabic graph associated to the decorated permutation $\{3,6,8,5,9,7,11,10,13\}$ in $\mathrm{G}_{+}(3,9)$.

Altogether, we therefore encounter a total of 25 algebraic factors associated to this cell of $\mathrm{G}_{+}(3,9)$. We find that 20 of these 25 factors are multiplicatively independent; including, of course, the $11 \sqrt{\Delta_{3579}}$-containing algebraic letters that appear in the 2-loop nine-particle NMHV amplitude. The additional algebraic letters that we find may appear in higher, not yet computed nine-particle amplitudes, or they may be analogs of the "non-cluster variable" rational quantities that generally appear when solving $C Z=0$ for non-top cells (see $[13,14]$ for examples).

\section{Acknowledgments}

We are grateful to L. Ren for collaboration on the related work [17] and to N. Arkani-Hamed for numerous encouraging conversations. This work was supported in part by the US Department of Energy under contract DE-SC0010010 Task A and by Simons Investigator Award \#376208 (AV). Plabic graphs were drawn with the help of [26].

Open Access. This article is distributed under the terms of the Creative Commons Attribution License (CC-BY 4.0), which permits any use, distribution and reproduction in any medium, provided the original author(s) and source are credited. 


\section{References}

[1] S. Fomin and A. Zelevinsky, Cluster algebras I: Foundations, J. Am. Math. Soc. 15 (2002) 497 [math/0104151].

[2] S. Fomin and A. Zelevinsky, Cluster algebras II: Finite type classification, Invent. Math. 154 (2003) 63 [math/0208229].

[3] J.S. Scott, Grassmannians and Cluster Algebras, Proc. Lond. Math. Soc. 92 (2006) 345 [math/0311149].

[4] S. Fomin, L. Williams and A. Zelevinsky, Introduction to Cluster Algebras. Chapters 1-3, arXiv: 1608.05735.

[5] S. Fomin, L. Williams and A. Zelevinsky, Introduction to Cluster Algebras. Chapters 4-5, arXiv: 1707.07190.

[6] S. Fomin, L. Williams and A. Zelevinsky, Introduction to Cluster Algebras. Chapter 6, arXiv:2008.09189.

[7] A.B. Goncharov, M. Spradlin, C. Vergu and A. Volovich, Classical Polylogarithms for Amplitudes and Wilson Loops, Phys. Rev. Lett. 105 (2010) 151605 [arXiv:1006.5703] [INSPIRE].

[8] J. Golden, A.B. Goncharov, M. Spradlin, C. Vergu and A. Volovich, Motivic Amplitudes and Cluster Coordinates, JHEP 01 (2014) 091 [arXiv: 1305.1617] [INSPIRE].

[9] S. Caron-Huot et al., The Steinmann Cluster Bootstrap for $N=4$ Super Yang-Mills Amplitudes, PoS CORFU2019 (2020) 003 [arXiv: 2005.06735] [INSPIRE].

[10] N. Arkani-Hamed, T. Lam and M. Spradlin, Non-perturbative geometries for planar $\mathcal{N}=4$ SYM amplitudes, JHEP 03 (2021) 065 [arXiv: 1912.08222] [INSPIRE].

[11] J. Drummond, J. Foster, O. Gürdogan and C. Kalousios, Algebraic singularities of scattering amplitudes from tropical geometry, JHEP 04 (2021) 002 [arXiv: 1912.08217] [INSPIRE].

[12] N. Henke and G. Papathanasiou, How tropical are seven- and eight-particle amplitudes?, JHEP 08 (2020) 005 [arXiv: 1912.08254] [INSPIRE].

[13] J. Mago, A. Schreiber, M. Spradlin and A. Volovich, Symbol alphabets from plabic graphs, JHEP 10 (2020) 128 [arXiv:2007.00646] [INSPIRE].

[14] S. He and Z. Li, A note on letters of Yangian invariants, JHEP 02 (2021) 155 [arXiv:2007.01574] [INSPIRE].

[15] J. Mago, A. Schreiber, M. Spradlin, A. Yelleshpur Srikant and A. Volovich, Symbol alphabets from plabic graphs II: rational letters, JHEP 04 (2021) 056 [arXiv: 2012.15812] [INSPIRE].

[16] A. Herderschee, Algebraic branch points at all loop orders from positive kinematics and wall crossing, JHEP 07 (2021) 049 [arXiv:2102.03611] [INSPIRE].

[17] L. Ren, M. Spradlin and A. Volovich, Symbol Alphabets from Tensor Diagrams, arXiv:2106.01405 [INSPIRE].

[18] N. Henke and G. Papathanasiou, Singularities of eight-and nine-particle amplitudes from cluster algebras and tropical geometry, arXiv:2106.01392 [INSPIRE].

[19] N. Arkani-Hamed, J.L. Bourjaily, F. Cachazo, A.B. Goncharov, A. Postnikov and J. Trnka, Grassmannian Geometry of Scattering Amplitudes, Cambridge University Press (2016) [DOI] [arXiv:1212.5605] [INSPIRE]. 
[20] A. Postnikov, Total positivity, Grassmannians, and networks, math/0609764 [INSPIRE].

[21] N. Arkani-Hamed, F. Cachazo, C. Cheung and J. Kaplan, A Duality For The S Matrix, JHEP 03 (2010) 020 [arXiv:0907.5418] [InSPIRE].

[22] L.J. Mason and D. Skinner, Dual Superconformal Invariance, Momentum Twistors and Grassmannians, JHEP 11 (2009) 045 [arXiv: 0909. 0250] [INSPIRE].

[23] S. He, Z. Li and C. Zhang, Two-loop octagons, algebraic letters and $\bar{Q}$ equations, Phys. Rev. D 101 (2020) 061701 [arXiv: 1911.01290] [INSPIRE].

[24] S. He, Z. Li and C. Zhang, The symbol and alphabet of two-loop NMHV amplitudes from $\bar{Q}$ equations, JHEP 03 (2021) 278 [arXiv: 2009.11471] [INSPIRE].

[25] M. Gekhtman, M.Z. Shapiro and A.D. Vainshtein, Cluster algebras and Poisson geometry, Mosc. Math. J. 3 (2003) 899 [math/0208033].

[26] J.L. Bourjaily, Positroids, Plabic Graphs, and Scattering Amplitudes in Mathematica, arXiv:1212.6974 [INSPIRE]. 